\title{
Exponential mixing for 2D Navier-Stokes equations perturbed by an unbounded noise
}

\author{
Armen Shirikyan \\ Laboratoire de Mathématiques \\ Université de Paris-Sud XI, Bâtiment 425 \\ 91405 Orsay Cedex, France \\ E-mail: Armen.Shirikyan@math.u-psud.fr
}

\begin{abstract}
The paper is devoted to the problem of mixing for two-dimensional Navier-Stokes equations perturbed by an unbounded kick force. We develop the coupling approach suggested in [16] to show that any solution exponentially converges to the stationary measure in the dual Lipschitz norm. This property complements some earlier results established in [15] for the same model.
\end{abstract}

AMS subject classifications: 35Q30, 60H15, 60J05

Keywords: Navier-Stokes equations, exponential mixing, coupling.

\section{Contents}

$\begin{array}{lll}0 & \text { Introduction } & 2\end{array}$

1 Preliminaries 5

1.1 Functional spaces and Markov chains associated with the NS system 5

1.2 Estimates for some stopping times . . . . . . . . . . . . . 7

1.3 Foiaş-Prodi type estimate . . . . . . . . . . . . . . . . 11

2 Main result 11

3 Coupling operator and its properties 14

4 Proof of Proposition 2.3 16

4.1 Description of coupled sequences . . . . . . . . . . . . 16

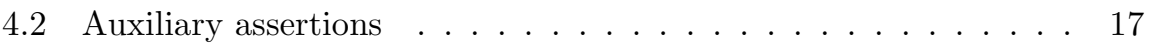

4.3 Proof of $(2.8)-(2.12) \ldots \ldots . \ldots . . \ldots 20$ 
5.1 Proof of Proposition $1.4 \ldots \ldots \ldots \ldots \ldots$

5.2 An estimate for the hitting time of a large ball . . . . . . . . 23

5.3 A property of maximal coupling . . . . . . . . . . . . . . 24

Bibliography

\section{Introduction}

Let $D \subset \mathbb{R}^{2}$ be a bounded domain with smooth boundary $\partial D$. We consider the Navier-Stokes (NS) system

$$
\dot{u}-\nu \Delta u+(u, \nabla) u+\nabla p=\eta(t, x), \quad \operatorname{div} u=0, \quad x \in D,
$$

where $\nu>0$ is the viscosity, $u=u(t, x)$ is the velocity field, $p=p(t, x)$ is the pressure, and $\eta(t, x)$ is an external force. Equation (0.1) is supplemented with Dirichlet boundary condition

$$
\left.u\right|_{\partial D}=0 .
$$

It is assumed that the right-hand side of (0.1) is a random process of the form

$$
\eta(t, x)=\sum_{k=1}^{\infty} \eta_{k}(x) \delta(t-k),
$$

where $\delta(t)$ is the Dirac measure and $\eta_{k}$ are independent identically distributed (i.i.d.) random variables with range in the space $L^{2}\left(D, \mathbb{R}^{2}\right)$ of square-integrable vector fields on $D$. (See Section 2 for the exact conditions on $\eta_{k}$.)

As was shown in $[14,15]$, the long-time behaviour of solutions for Eqs. (0.1) (0.3) can be described in terms of the following random dynamical system:

$$
u_{k}=S_{1}\left(u_{k-1}\right)+\eta_{k}, \quad k \geq 1,
$$

where $u_{k}=u(k, x)$ and $S_{t}$ is the resolving semi-group for the deterministic NS system $(0.1),(0.2)$ with $\eta \equiv 0$. It is proved in $[14,15]$ that if the distribution of $\eta_{k}$ is sufficiently non-degenerate, then there is a unique stationary measure for (0.4). Moreover, the coupling approach suggested in $[16,17,12]$ enabled one to establish exponential convergence of solutions for $(0.1),(0.2)$ to the stationary measure on condition that the support of distribution of $\eta_{k}$ is bounded. The aim of this article is to show that a similar result holds in the general case.

The problem of ergodicity for randomly forced two-dimensional NS system and other parabolic PDE's with different types of additive noise was intensively studied beginning with the paper [8] by Flandoli and Maslowski in 1995 (see [14, $2,15,16,17,19,12]$ for discrete forcing and $[6,3,7,20,11,18]$ for white noise). We refer the reader to $[18,13]$ for a detailed discussion of the results and methods developed since then.

In this paper, we combine the coupling used in [16] with a stopping time technique that is often applied to establish exponential mixing for processes 
with unbounded phase space (see $[1,26]$ ). Without going into details, let us describe the main ideas. ${ }^{1}$

Let $P_{k}(u, \Gamma)$ be the transition function for a family of Markov chains in a Hilbert space $H$ endowed with norm $|\cdot|$. Suppose that we can construct a probability space $\Omega$ and a family Markov chains $\left(U_{k}, \mathbb{P}_{U}\right), U_{k}=\left(u_{k}, u_{k}^{\prime}\right)$, in the direct product $\mathbf{H}=H \times H$ such that the distributions of $u_{k}$ and $u_{k}^{\prime}$ under the law $\mathbb{P}_{U}, U=\left(u, u^{\prime}\right)$, coincide with $P_{k}(u, \cdot)$ and $P_{k}\left(u^{\prime}, \cdot\right)$, respectively, and the following two properties hold:

(i) Let $\sigma=\min \left\{k \geq 1:\left|u_{k}-u_{k}^{\prime}\right|>e^{-2 k}\right\}$, where the minimum over an empty set is $+\infty$. Then there is a subset $\mathbf{B} \subset \mathbf{H}$ and a constant $C>0$ such that, for $U=\left(u, u^{\prime}\right) \in \mathbf{B}$, we have

$$
\mathbb{P}_{U}\{\sigma=+\infty\} \geq \frac{1}{2}, \quad \mathbb{P}_{U}\{\sigma=k\} \leq C e^{-k} .
$$

(ii) Let $\tau=\min \left\{k \geq 0: U_{k} \in \mathbf{B}\right\}$. Then there is $\gamma>0$ such that

$$
\mathbb{E}_{U} e^{\gamma \tau}<\infty \text { for any } U \in \mathbf{H} \text {. }
$$

In this case, the difference $P_{k}(u, \cdot)-P_{k}\left(u^{\prime}, \cdot\right)$, regarded as a signed measure in $H$, goes to zero in the dual Lipschitz norm $\|\cdot\|_{\mathcal{L}}^{*}$ exponentially fast. (See Notation for the definition of $\|\cdot\|_{\mathcal{L}}^{*}$.) Indeed, it follows from (i) that, each time the process is in $\mathbf{B}$, with probability $\geq \frac{1}{2}$ we have $\sigma=+\infty$, which means that the difference $\Delta_{k}=\left|u_{k}-u_{k}^{\prime}\right|$ goes to zero exponentially fast. Let us consider a sequence of stopping times $\rho_{k}$ defined by the following rule. Let $\rho_{0}$ be the first hitting time of $\mathbf{B}$ (i.e., $\rho_{0}=\tau$ ). With probability $\geq \frac{1}{2}$, we have $\sigma=+\infty$ for the chain starting from $U_{\rho_{0}}$, and in this case we set $\rho_{k}=+\infty$ for $k \geq 2$. Otherwise we denote by $\rho$ the first instant after $\sigma$ when $U_{\rho_{0}+k}$ hits $\mathbf{B}$ and define $\rho_{1}$ by the formula $\rho_{1}=\rho_{0}+\rho$. In general, if $\rho_{k}$ is already defined, then $\rho_{k+1}=\rho_{k}+\rho$, where $\rho$ is the first instant after $\sigma$ when the chain starting from $U_{\rho_{k}}$ hits $\mathbf{B}$. As in the case of $\rho_{0}$, with probability $\geq \frac{1}{2}$ we have $\rho_{l}=+\infty$ for $l \geq k+1$.

The above construction implies that, if $\rho_{k}<+\infty$ and $\rho_{k+1}=+\infty$, then $\Delta_{\rho_{k}+m} \leq e^{-2 m}$ for all $m \geq 0$. Using the strong Markov property (SMP) and assertions (i) and (ii), it can be shown that $\mathbb{P}_{U}\left\{\rho_{k}<+\infty\right\} \leq 2^{-k}$. What has been said implies that with probability $\geq 1-2^{-k-1}$ we have

$$
\left|u_{k}-u_{k}^{\prime}\right| \leq e^{-2 k+\rho_{k}} \quad \text { for all } k \geq \rho_{k} .
$$

Moreover, further analysis enables one to show that

$$
\mathbb{P}_{U}\left\{k \leq \rho_{k}<\infty\right\} \leq C e^{-\alpha k},
$$

where $C$ and $\alpha$ are positive constants. Combining (0.5) and (0.6), we see that

$$
\mathbb{P}_{U}\left\{\left|u_{k}-u_{k}^{\prime}\right|>e^{-k}\right\} \leq 2^{-k-1}+C e^{-\alpha k} \quad \text { for } \quad k \geq 1 .
$$

\footnotetext{
${ }^{1}$ We partially follow explanations in [26, Section 14$]$.
} 
Thus, the difference $\left|u_{k}-u_{k}^{\prime}\right|$ converges to zero in probability exponentially fast. As in the case of bounded kicks, this implies the required assertion (cf. Lemma 1.3 in $[16]$ ).

We note that the scheme described above is not entirely accurate. In particular, the definition of the stopping time $\sigma$ used in the main text is different (see Section 4.2). However, properties (i) and (ii) form the main mechanism that leads to exponential convergence. We shall show in fact that $\left|u_{k}-u_{k}^{\prime}\right|$ goes to zero exponentially fast with probability 1 (see Corollary 2.4).

We also note that, in the context of randomly forced PDE's, some ideas related to the above approach were used earlier by Mattingly [20] and Hairer [11]. However, their schemes (which are rather complicated) are based on a factorisation of the transition function and introduction of an auxiliary family of Markov chains that takes into account the "future" of the original family.

The paper is organised as follows. In Section 1, some preliminaries are compiled. In Section 2, we formulate the main result of this paper and show that it can be derived from the existence of a coupling of solutions. Section 3 is devoted to studying a coupling operator introduced in [16]. In Section 4, we describe the aforementioned coupling for solutions and show that it possesses all necessary properties. The Appendix contains proof of some auxiliary assertions.

\section{Notation}

For a bounded domain $D \subset \mathbb{R}^{2}$, we denote by $L^{2}\left(D, \mathbb{R}^{2}\right)$ the space of square integrable vector functions $\left(u_{1}, u_{2}\right)$, by $|\cdot|$ the natural norm in $L^{2}\left(D, \mathbb{R}^{2}\right)$, and by $H^{1}\left(D, \mathbb{R}^{2}\right)$ the Sobolev space of order 1 . Let $C_{0}^{\infty}(D)$ be the space of infinitely smooth functions on $D$ with compact support and let $\mathcal{V}$ be the space of vector functions $u=\left(u_{1}, u_{2}\right)$ such that $u_{i} \in C_{0}^{\infty}(D)$ for $i=1,2$ and $\operatorname{div} u=0$. We denote by $H$ and $V$ the closures of $\mathcal{V}$ in the spaces $L^{2}\left(D, \mathbb{R}^{2}\right)$ and $H^{1}\left(D, \mathbb{R}^{2}\right)$, respectively. In what follows, we assume that the domain $D$ is fixed and do not specify the dependence of various constants on $D$.

Let $\Pi$ be the orthogonal projection in $L^{2}\left(D, \mathbb{R}^{2}\right)$ onto the space $H$ and let $L$ be the Stokes operator defined as the restriction of $-\Pi \Delta$ to the space $H$ with natural domain of definition. It is well known $[25,4]$ that $L$ is a selfadjoint operator with discrete spectrum. Let $\left\{e_{1}, e_{2}, \ldots\right\}$ be a complete set of normalised eigenfunctions for $L$ that correspond to eigenvalues $\alpha_{1} \leq \alpha_{2} \leq \cdots$. We denote by $H_{N} \subset H$ the linear span of $\left\{e_{1}, \ldots, e_{N}\right\}$ and by $H_{N}^{\perp}$ its orthogonal complement in $H$. Let $\mathrm{P}_{N}$ and $\mathrm{Q}_{N}$ be the orthogonal projections onto $H_{N}$ and $H_{N}^{\perp}$, respectively.

Let $\mathcal{B}(H)$ be the Borel $\sigma$-algebra in $H$ and let $\mathcal{P}(H)$ be the family of all probability measures on $(H, \mathcal{B}(H))$. If $f(u)$ is an integrable function on $H$ and $\mu \in \mathcal{P}(H)$, then $(\mu, f)$ denotes the integral of $f$ over $H$ with respect to $\mu$. Let $\mathcal{L}(H)$ be the space of functions $f: H \rightarrow \mathbb{R}$ such that

$$
\|f\|_{\mathcal{L}}:=\sup _{u \in H}|f(u)|+\sup _{u, v \in H} \frac{|f(u)-f(v)|}{|u-v|}<\infty .
$$


We shall use the following metric on the space $\mathcal{P}(H)$ (see [5, Section 11.8]:

$$
\left\|\mu_{1}-\mu_{2}\right\|_{\mathcal{L}}^{*}:=\sup _{\|f\|_{\mathcal{L}} \leq 1}\left|\left(\mu_{1}, f\right)-\left(\mu_{2}, f\right)\right|, \quad \mu_{1}, \mu_{2} \in \mathcal{P}(H) .
$$

We denote by $\mathcal{P}_{1}(H)$ the set of measures $\mu \in \mathcal{P}(H)$ for which

$$
\mathfrak{m}_{1}(\mu):=\int_{H}|u| \mu(d u)<\infty .
$$

Let $(\Omega, \mathcal{F})$ be a measurable space. For set $A \in \mathcal{F}$, we denote by $A^{c}$ its complement and by $I(A)$ or $I_{A}$ its indicator function. If $\xi$ is a random variable, then $\mathcal{D}(\xi)$ stands for its distribution.

If $u_{k}$ is a sequence, and we let $k=T_{n}$, then we shall sometimes write $u\left(T_{n}\right)$ to avoid double subscript. We denote by $C$ various unessential constants and by $a \wedge b(a \vee b)$ the minimum (maximum) of $a$ and $b$.

\section{Preliminaries}

In this section, we have compiled some auxiliary assertions that will be used in the proof of the main result. To simplify notation, in what follows we assume that $\nu=1$. All the results remain valid for $0<\nu<1$.

\subsection{Functional spaces and Markov chains associated with the NS system}

Let $S_{t}: H \rightarrow H$ be the resolving semi-group for the NS system with $\eta \equiv 0$ and let $S=S_{1}$. We shall need the following two inequalities for $S_{t}$, which can be obtained by taking the scalar product in $H$ of the NS system and the solution $u(t)$ (e.g., see $[25,4]$ or $[15$, Section 6$])$ :

$$
|S(u)| \leq q|u|, \quad 2 \int_{0}^{1}\left\|S_{t}(u)\right\|^{2} d t \leq|u|^{2},
$$

where $\|v\|=\left|L^{\frac{1}{2}} v\right|$ is the norm in $V, q=e^{-\alpha_{1}}<1$ and $u$ is any function in $H$.

Let us consider the random dynamical system

$$
u_{k}=S\left(u_{k-1}\right)+\eta_{k},
$$

where $k \geq 1$ and $\left\{\eta_{k}\right\}$ is a sequence of i.i.d. random variables in $H$ defined on a probability space $(\Omega, \mathcal{F}, \mathbb{P})$ and satisfying the following inequality with a constant $\varkappa>0$ :

$$
Q:=\mathbb{E} \exp \left(\varkappa\left|\eta_{1}\right|^{2}\right)<\infty .
$$

We study Eq. (1.2) supplemented with the initial condition

$$
u_{0}=u,
$$

where $u=u(x)$ is a random variable in $H$ independent of $\eta_{k}, k \geq 1$. The following result is a simple consequence of the first inequality in (1.1). 
Proposition 1.1. Let $u_{k}, k \geq 0$, be the random sequence defined by (1.2), (1.4). Then for any $m \geq 1$ the following statements hold:

(i) There is a constant $C_{m}>0$ such that

$$
\left|u_{k}\right|^{m} \leq q^{k}|u|^{m}+C_{m} \sum_{l=1}^{k} q^{k-l}\left|\eta_{l}\right|^{m}, \quad k \geq 1 .
$$

Furthermore, we have $C_{1}=1$ and $C_{2}=(1-q)^{-1}$.

(ii) Let $K_{m}=\mathbb{E}\left|\eta_{1}\right|^{m}$. If $\mathbb{E}|u|^{m}<\infty$, then

$$
\mathbb{E}\left|u_{k}\right|^{m} \leq \mathbb{E}\left(q^{k}|u|^{m}\right)+(1-q)^{-1} C_{m} K_{m}, \quad k \geq 1 .
$$

Moreover, if $\sigma$ is a stopping time such that $E=\sum_{l=1}^{\infty}(\mathbb{P}\{\sigma=l\})^{\frac{1}{2}}<\infty$, then

$$
\mathbb{E}\left(I_{\{\sigma<\infty\}}\left|u_{\sigma}\right|^{m}\right) \leq \mathbb{E}\left(q^{\sigma}|u|^{m}\right)+\frac{E C_{m} K_{2 m}^{\frac{1}{2}}}{1-q}
$$

Proof. Inequality (1.5) follows from [15, Section 6.1] and (1.6) is easily derived from (1.5) by taking the expectation. Therefore we confine ourselves to the proof of (1.7).

Let us multiply (1.5) by $I_{\{\sigma<\infty\}}$ and set $k=\sigma \wedge n$, where $n \geq 1$ is an integer. This results in

$$
\begin{aligned}
I_{\{\sigma<\infty\}}\left|u_{\sigma \wedge n}\right|^{m} & \leq I_{\{\sigma<\infty\}} q^{\sigma \wedge n}|u|^{m}+C_{m} I_{\{\sigma<\infty\}} \sum_{l=1}^{\sigma \wedge n} q^{\sigma \wedge n-l}\left|\eta_{l}\right|^{m} \\
& \leq q^{\sigma \wedge n}|u|^{m}+C_{m} \sum_{s=1}^{\infty} \sum_{l=1}^{s \wedge n} q^{s \wedge n-l} I_{\{\sigma=s\}}\left|\eta_{l}\right|^{m} .
\end{aligned}
$$

Taking the expectation and using Fatou's lemma and the monotone convergence theorem to pass to the limit as $n \rightarrow \infty$ in the above inequality, we derive

$$
\mathbb{E}\left(I_{\{\sigma<\infty\}}\left|u_{\sigma}\right|^{m}\right) \leq \mathbb{E}\left(q^{\sigma}|u|^{m}\right)+C_{m} \mathcal{E}_{m},
$$

where

$$
\mathcal{E}_{m}=\sum_{s=1}^{\infty} \sum_{l=1}^{s} q^{s-l} \mathbb{E}\left(I_{\{\sigma=s\}}\left|\eta_{l}\right|^{m}\right)
$$

We now note that

$$
\mathcal{E}_{m} \leq \sum_{l=1}^{\infty} \sum_{s=l}^{\infty} q^{s-l}\left(\mathbb{E}\left|\eta_{l}\right|^{2 m}\right)^{\frac{1}{2}}(\mathbb{P}\{\sigma=l\})^{\frac{1}{2}} \leq E K_{2 m}^{\frac{1}{2}}(1-q)^{-1} .
$$

Substituting this inequality into (1.8), we obtain (1.7). 
Proposition 1.1 enables one to construct a family of Markov chains associated with (1.2) and parametrised by the initial condition $u$ from (1.4). For the reader's convenience, we now briefly recall the construction.

Let $\mathcal{F}_{k}$ be the $\sigma$-algebra generated by $\eta_{l}, 1 \leq l \leq k$. We set

$$
\Omega^{\prime}=H \times \Omega, \quad \mathcal{F}^{\prime}=\mathcal{B}(H) \otimes \mathcal{F}, \quad \mathcal{F}_{k}^{\prime}=\mathcal{B}(H) \otimes \mathcal{F}_{k}, \quad \mathbb{P}_{u}=\delta_{u} \otimes \mathbb{P},
$$

where $\mathcal{B}(H)$ is the Borel $\sigma$-algebra on $H, \delta_{u} \in \mathcal{P}(H)$ is the Dirac measure concentrated at $u$, and $\otimes$ denotes the direct product for measures and $\sigma$-algebras. For $\omega^{\prime}=(u, \omega) \in \Omega^{\prime}$, let $u_{k}^{\prime}\left(\omega^{\prime}\right)=u_{k}(u, \omega)$, where $u_{k}(u, \omega)$ is the random sequence defined by $(1.2),(1.4)$. Since $\eta_{k}$ are i.i.d., $\left(u_{k}^{\prime}, \mathbb{P}_{u}\right)$ is a family of homogeneous Markov chains with respect to $\mathcal{F}_{k}^{\prime}$. Its transition function is given by the formula $P_{k}(u, \Gamma)=\mathbb{P}_{u}\left\{u_{k}^{\prime} \in \Gamma\right\}$, and the corresponding Markov operators have the form

$$
\mathfrak{P}_{k} f(u)=\int_{H} P_{k}(u, d v) f(v), \quad \mathfrak{P}_{k}^{*} \mu(\Gamma)=\int_{H} P_{k}(u, \Gamma) \mu(d u),
$$

where $f: H \rightarrow \mathbb{R}$ is a bounded continuous function and $\mu \in \mathcal{P}(H)$. In what follows, we shall drop the prime from notation and write $\omega, u_{k}, \Omega, \mathcal{F}, \mathcal{F}_{k}$ instead of $\omega^{\prime}, u_{k}^{\prime}, \Omega^{\prime}, \mathcal{F}^{\prime}, \mathcal{F}_{k}^{\prime}$. This will not lead to confusion.

\subsection{Estimates for some stopping times}

For any sequence $v_{k} \in H, l \leq k \leq m$, we set

$$
\left\langle\|\boldsymbol{v}\|^{2}\right\rangle_{l}^{m}:=\frac{1}{m-l+1} \sum_{k=l}^{m} \int_{0}^{1}\left\|S_{t}\left(v_{k}\right)\right\|^{2} d t
$$

Let us introduce the stopping time

$$
T(M)=\min \left\{k \geq 1:\left\langle\|\boldsymbol{u}\|^{2}\right\rangle_{0}^{k}>M\right\},
$$

where $M>0$ is a constant, $\left(u_{k}, \mathbb{P}_{u}\right)$ is the Markov chain defined in the foregoing subsection, and we set $T(M)=\infty$ if the condition in the brackets is never satisfied.

For any probability measure $\lambda \in \mathcal{P}(H)$, we set

$$
\mathbb{P}_{\lambda}(A)=\int_{\Omega} \mathbb{P}_{u}(A) \lambda(d u), \quad A \in \mathcal{F} .
$$

Note that $\mathbb{P}_{\lambda}$ is a probability measure on $(\Omega, \mathcal{F})$, and $u_{k}$ is a Markov chain with respect to $\mathbb{P}_{\lambda}$ (see $[22$, Section 1.2$\left.]\right)$.

Proposition 1.2. Suppose that (1.3) holds. Then there is $\delta>0$ depending only on $\varkappa$ such that for any initial measure $\lambda \in \mathcal{P}(H)$ satisfying the condition

$$
\int_{H} e^{\delta|u|^{2}} \lambda(d u) \leq R
$$


where $R>0$ is a constant, we have

$$
\mathbb{P}_{\lambda}\{T(M)=k\} \leq R Q^{k} e^{-\delta M(k+1)}, \quad k \geq 1 .
$$

In particular, if $\delta M \geq \log (R+Q)$, then

$$
\mathbb{P}_{\lambda}\{T(M)=\infty\} \geq \frac{R}{R+Q}>0 .
$$

The proof will imply that (1.11) is valid for $0<\delta \leq \delta_{0}$, where $\delta_{0}>0$ depends only on $\varkappa$.

Proof. Inequality (1.12) follows easily from (1.11), and therefore we confine ourselves to the proof of (1.11).

As is shown in [15, formula (1.24)], for any integer $k \geq 0$ we have ${ }^{2}$

$$
\left|u_{k}\right|^{2}+k\left\langle\|\boldsymbol{u}\|^{2}\right\rangle_{0}^{k-1} \leq\left|u_{0}\right|^{2}+\left(1+\alpha_{1}^{-1}\right) \sum_{l=1}^{k}\left|\eta_{l}\right|^{2} .
$$

Setting $u=u_{k}$ in the second inequality in (1.1) and adding the resulting estimate to $(1.13)$, we obtain

$$
I_{k}:=(k+1)\left\langle\|\boldsymbol{u}\|^{2}\right\rangle_{0}^{k} \leq\left|u_{0}\right|^{2}+\left(1+\alpha_{1}^{-1}\right) \sum_{l=1}^{k}\left|\eta_{l}\right|^{2} .
$$

Let $\delta$ be such that $\delta\left(1+\alpha_{1}^{-1}\right) \leq \varkappa$. Then it follows from (1.3) and (1.14) that

$$
\mathbb{E}_{\lambda} e^{\delta I_{k}} \leq R Q^{k}
$$

Chebyshev's inequality now implies that

$$
\mathbb{P}_{\lambda}\left\{I_{k}>M(k+1)\right\} \leq R Q^{k} e^{-\delta M(k+1)},
$$

It remains to note that $\{T(M)=k\} \subset\left\{I_{k}>M(k+1)\right\}$.

For any $d>0$, we define the first hitting time of the ball $B_{d}=\{|u| \leq d\} \subset H$ by the formula

$$
\tau_{d}=\min \left\{k \geq 0:\left|u_{k}\right| \leq d\right\} .
$$

Proposition 1.3. Suppose that $K=\mathbb{E}\left|\eta_{1}\right|<\infty$ and $\mathbb{P}\left\{\left|\eta_{1}\right| \leq \varepsilon\right\}>0$ for any $\varepsilon>0$. Then for any $d>0$ there are positive constants $\gamma$ and $C$ such that for any initial measure $\lambda \in \mathcal{P}_{1}(H)$ we have

$$
\mathbb{E}_{\lambda} e^{\gamma \tau_{d}} \leq C\left(1+\mathfrak{m}_{1}(\lambda)\right)
$$

Proof. The proof is based on the existence of a Lyapunov function and the fact that any trajectory visits arbitrarily small neighbourhood of zero with positive probability. More precisely, we use the following two observations:

\footnotetext{
${ }^{2}$ In [15], the case of a torus is discussed. However, the derivation of formula (1.24) remains valid for the case of Dirichlet boundary condition.
} 
(a) There are positive constants $R$ and $C$ such that, for any initial measure $\lambda \in \mathcal{P}_{1}(H)$, we have $\tau_{R}<\infty \mathbb{P}_{\lambda}$-a.s. and

$$
\mathbb{E}_{\lambda} q^{-\tau_{R}} \leq C\left(1+\mathfrak{m}_{1}(\lambda)\right) .
$$

(b) For any $R>0$ and $d>0$ there is an integer $l \geq 1$ and a constant $p>0$ such that

$$
\mathbb{P}_{u}\left\{\left|u_{l}\right| \leq d\right\} \geq p \quad \text { for any } \quad u \in H, \quad|u| \leq R .
$$

Taking these properties for granted, let us complete the proof of the proposition. We follow a standard argument (see [10, Sections III.7 and IV.2] and [26, Section 13]).

Step 1. Without loss of generality, we can assume that, on the probability space $\Omega$, there is defined a shift operator $\theta_{h}: \Omega \rightarrow \Omega$ (where $h \geq 0$ is an integer) such that $u_{k}\left(\theta_{h} \omega\right)=u_{k+h}(\omega)$; see [22, Section 1] for details.

We fix a constant $R>0$ for which (1.17) holds and define the stopping time $T=\tau+l$, where $l=l(R, d) \geq 1$ is the integer in statement (b) and $\tau:=\tau_{R}$. Let us consider iterations of $T$ :

$$
T_{0}=T, \quad T_{m}=T_{m-1}+T \circ \theta_{T_{m-1}}, \quad m \geq 1 .
$$

For any initial measure $\lambda \in \mathcal{P}_{1}(H)$, we set

$$
P_{\lambda}(m)=\mathbb{P}_{\lambda}\left(\bigcap_{n=1}^{m}\left\{\left|u\left(T_{n}\right)\right|>d\right\}\right) .
$$

We claim that

$$
P_{\lambda}(m) \leq(1-p)^{m}, \quad m \geq 1 .
$$

Indeed, let us set $T_{m}^{\prime}=T_{m-1}+\tau \circ \theta_{T_{m-1}}$ and note that $T_{m}=T_{m}^{\prime}+l$. By the strong Markov property (SMP), it follows that

$$
\mathbb{P}_{\lambda}\left\{\left|u\left(T_{m}\right)\right|>d \mid \mathcal{F}_{T_{m}^{\prime}}\right\}=\mathbb{P}_{u\left(T_{m}^{\prime}\right)}\left\{\left|u_{l}\right|>d\right\} \leq 1-p,
$$

where we used inequality (1.18) and the fact that $\left|u\left(T_{m}^{\prime}\right)\right| \leq R$. Therefore, using again the SMP, we derive

$P_{\lambda}(m)=\mathbb{E}_{\lambda}\left(\prod_{n=1}^{m-1} I\left(\left|u\left(T_{n}\right)\right|>d\right) \mathbb{P}_{\lambda}\left\{\left|u\left(T_{m}\right)\right|>d \mid \mathcal{F}_{T_{m}^{\prime}}\right\}\right) \leq(1-p) P_{\lambda}(m-1)$.

Iteration of this inequality results in (1.19).

Step 2. We now show that

$$
\mathbb{E}_{\lambda} q^{-T_{m}} \leq C_{1} D^{m}\left(1+\mathfrak{m}_{1}(\lambda)\right), \quad m \geq 1,
$$


where the positive constants $C_{1}$ and $D$ depend only on $d$. Indeed, let us set $T^{\prime}=l+\tau \circ \theta_{l}$ and note that, by the SMP and inequalities (1.17) and (1.6) (with $k=l$ and $m=1$ ), for any $u \in B_{R}$ we have

$$
\mathbb{E}_{u} q^{-T^{\prime}}=q^{-l} \mathbb{E}_{u}\left\{\mathbb{E}_{u_{l}}\left(q^{-\tau}\right)\right\} \leq C q^{-l} \mathbb{E}_{u}\left(1+\left|u_{l}\right|\right) \leq D,
$$

where $D>0$ does not depend of $u$. Furthermore, the stopping times $T_{m}^{\prime}$ introduced above can be represented in the form

$$
T_{0}^{\prime}=\tau, \quad T_{m}^{\prime}=T_{m-1}^{\prime}+T^{\prime} \circ \theta_{T_{m-1}^{\prime}}, \quad m \geq 1 .
$$

Therefore, using the SMP and inequality (1.21), for any $\lambda \in \mathcal{P}_{1}(H)$ we derive

$$
\mathbb{E}_{\lambda} q^{-T_{m}^{\prime}}=\mathbb{E}_{\lambda}\left\{q^{-T_{m-1}^{\prime}}\left(\mathbb{E}_{u\left(T_{m-1}^{\prime}\right)} q^{-T^{\prime}}\right)\right\} \leq D \mathbb{E}_{\lambda} q^{-T_{m-1}^{\prime}}
$$

Iteration of this estimate results in

$$
\mathbb{E}_{\lambda} q^{-T_{m}^{\prime}} \leq D^{m} \mathbb{E}_{\lambda} q^{-T_{0}^{\prime}} \leq C D^{m}\left(1+\mathfrak{m}_{1}(\lambda)\right),
$$

where we used (1.17). Recalling that $T_{m}=T_{m}^{\prime}+l$, we obtain inequality (1.20) with $C_{1}=C q^{-l}$.

Step 3. We can now prove (1.16). For any initial measure $\lambda \in \mathcal{P}_{1}(H)$ and any integer $m \geq 1$, we have

$$
\begin{aligned}
\mathbb{P}_{\lambda}\left\{\tau_{d} \geq k\right\} & =\mathbb{P}_{\lambda}\left\{\tau_{d} \geq k, T_{m}<k\right\}+\mathbb{P}_{\lambda}\left\{\tau_{d} \geq k, T_{m} \geq k\right\} \\
& \leq \mathbb{P}_{\lambda}\left\{\tau_{d}>T_{m}\right\}+\mathbb{P}_{\lambda}\left\{T_{m} \geq k\right\}
\end{aligned}
$$

It follows from (1.19), (1.20), and Chebyshev's inequality that

$$
\begin{aligned}
\mathbb{P}_{\lambda}\left\{\tau_{d}>T_{m}\right\} & \leq P_{\lambda}(m) \leq(1-p)^{m}, \\
\mathbb{P}_{\lambda}\left\{T_{m} \geq k\right\} & \leq q^{k} \mathbb{E}_{\lambda} q^{-T_{m}} \leq C_{1} D^{m} q^{k}\left(1+\mathfrak{m}_{1}(\lambda)\right) .
\end{aligned}
$$

Substitution of these estimates into (1.22) results in

$$
\mathbb{P}_{\lambda}\left\{\tau_{d} \geq k\right\} \leq(1-p)^{m}+C_{1} D^{m} q^{k}\left(1+\mathfrak{m}_{1}(\lambda)\right) .
$$

Choosing $m=[\varepsilon k]+1$, where $\varepsilon>0$ is sufficiently small and $[r]$ is the integer part of $r>0$, we obtain

$$
\mathbb{P}_{\lambda}\left\{\tau_{d} \geq k\right\} \leq C_{2} e^{-\alpha k}\left(1+\mathfrak{m}_{1}(\lambda)\right),
$$

where $C_{2}$ and $\alpha$ are some positive constants. Inequality (1.16) is an obvious consequence of (1.23).

Thus, it remains to establish assertions (a) and (b). The first of them is a consequence of Lemma 5.1 (see the Appendix). Assertion (b) follows immediately from the first inequality in (1.1) and the fact that $\mathbb{P}\left\{\left|\eta_{1}\right| \leq \varepsilon\right\}>0$ for any $\varepsilon>0$ (e.g., see [16, Lemma 3.1]). 


\subsection{Foiaş-Prodi type estimate}

We denote by $H_{N}$ the finite-dimensional subspace spanned by $\left\{e_{1}, e_{2}, \ldots, e_{N}\right\}$ and by $H_{N}^{\perp}$ its orthogonal complement in $H$. Let $\mathrm{P}_{N}$ and $\mathrm{Q}_{N}$ be the orthogonal projections onto $H_{N}$ and $H_{N}^{\perp}$, respectively.

For any two sequences $u_{k}, u_{k}^{\prime} \in H, l \leq k \leq m$, we set

$$
\left\langle\|\boldsymbol{u}\|^{2}+\left\|\boldsymbol{u}^{\prime}\right\|^{2}\right\rangle_{l}^{m}:=\left\langle\|\boldsymbol{u}\|^{2}\right\rangle_{l}^{m}+\left\langle\left\|\boldsymbol{u}^{\prime}\right\|^{2}\right\rangle_{l}^{m} .
$$

Let $l \leq m$ be some integers and let $\zeta_{k}$ and $\zeta_{k}^{\prime}, l+1 \leq k \leq m$, be two sequences in $H$. Suppose that sequences $u_{k}, u_{k}^{\prime} \in H$ satisfy the equations

$$
u_{k}=S\left(u_{k-1}\right)+\zeta_{k}, \quad u_{k}^{\prime}=S\left(u_{k-1}\right)+\zeta_{k}^{\prime}, \quad l+1 \leq k \leq m .
$$

The following result is an analogue of an estimate due to Foiaş and Prodi [9].

Proposition 1.4. Suppose that

$$
\mathrm{P}_{N} u_{k}=\mathrm{P}_{N} u_{k}^{\prime}, \quad \mathrm{Q}_{N} \zeta_{k}=\mathrm{Q}_{N} \zeta_{k}^{\prime} \quad \text { for } \quad l+1 \leq k \leq m .
$$

Then there is a constant $C>0$ not depending on $u_{k}, u_{k}^{\prime}, m, l$, and $N$ such that, for $l \leq k \leq m$, we have

$$
\left|u_{k}-u_{k}^{\prime}\right| \leq\left(C \alpha_{N+1}^{-\frac{1}{4}}\right)^{k-l} \exp \left(C(k-l)\left\langle\|\boldsymbol{u}\|^{2}+\left\|\boldsymbol{u}^{\prime}\right\|^{2}\right\rangle_{l}^{k-1}\right)\left|u_{l}-u_{l}^{\prime}\right| .
$$

In the case of a torus, the proposition is proved in [15] (see inequality (2.24)). For the Dirichlet boundary conditions, the proof is similar and is outlined in the Appendix (see Section 5.1).

\section{Main result}

To formulate the main result, we shall need the following hypothesis:

Condition 2.1. The i.i.d. random variables $\eta_{k}$ have the form

$$
\eta_{k}=\sum_{j=1}^{\infty} b_{j} \xi_{j k} e_{j}(x)
$$

where $\xi_{j k}$ are independent scalar random variables and $b_{j} \geq 0$ are some constants such that

$$
B:=\sum_{j=1}^{\infty} b_{j}^{2}<\infty .
$$

Moreover, for any $j \geq 1$ the distribution of $\xi_{j k}$ possesses a density $p_{j}(r)$ (with respect to the Lebesgue measure) that is a function of bounded variation satisfying the conditions

$$
\int_{\mathbb{R}} e^{r^{2}} p_{j}(r) d r \leq Q, \quad p_{j}(r)>0 \quad \text { for all } \quad r \in \mathbb{R},
$$

where $Q>0$ is constant not depending on $j$. 
Before formulating the main result of this paper, let us note that inequalities (2.1) and (2.2) imply that (1.3) holds with $\varkappa=\frac{1}{B}$ and that $\mathbb{P}\left\{\left|\eta_{1}\right|>\varepsilon\right\}>0$ for any $\varepsilon>0$. In particular, Propositions 1.1, 1.2, and 1.3 are valid if Condition 2.1 is satisfied.

We recall that a measure $\mu \in \mathcal{P}(H)$ is said to be stationary for the Markov semi-group $\mathfrak{P}_{k}^{*}$ if $\mathfrak{P}_{1}^{*} \mu=\mu$.

Theorem 2.2. Suppose that Condition 2.1 is satisfied. For any $B \geq 0$ there is an integer $N \geq 1$ such that, if

$$
b_{j} \neq 0 \quad \text { for } \quad j=1, \ldots, N
$$

then there is a unique stationary measure $\mu \in \mathcal{P}(H)$ for $\mathfrak{P}_{k}^{*}$. Moreover, there are positive constants $\beta$ and $C$ such that for any initial measure $\lambda \in \mathcal{P}_{1}(H)$ we have

$$
\left\|\mathfrak{P}_{k}^{*} \lambda-\mu\right\|_{\mathcal{L}}^{*} \leq C\left(1+\mathfrak{m}_{1}(\lambda)\right) e^{-\beta k}, \quad k \geq 1 .
$$

Theorem 2.2 will be derived from the proposition below, which is of independent interest. Before formulating it, let us explain its main idea.

The assertions of Theorem 2.2 concern the distributions of random sequences $\left\{u_{k}\right\}$ defined by (1.4) (and not the random sequences themselves). Suppose that $\zeta_{k}, k \geq 1$, is a sequence of i.i.d. in $H$ (which may be defined on a different probability space) such that $\mathcal{D}\left(\zeta_{k}\right)=\mathcal{D}\left(\eta_{k}\right)$ and let $\left\{v_{k}\right\}$ be a random sequence satisfying the relation $v_{k}=S\left(v_{k-1}\right)+\zeta_{k}$ for $k \geq 1$. In this case, if $\mathcal{D}\left(v_{0}\right)=\mathcal{D}\left(u_{0}\right)$ and $v_{0}$ is independent of $\left\{\zeta_{k}\right\}$, then $\mathcal{D}\left(v_{k}\right)=\mathcal{D}\left(u_{k}\right)$ for all $k$. Therefore, if the choice of $\zeta_{k}$ is such that $v_{k}$ converges in distribution, then the same is true for $u_{k}$.

Let us denote by $\mathbf{H}$ the direct product of two copies of $H$. In the proposition below, for any probability measure $\Lambda$ on $\mathbf{H}$ we construct a pair of random variables $\left(u_{0}, u_{0}^{\prime}\right)$ with distribution $\Lambda$ and two random sequences $\zeta_{k}$ and $\zeta_{k}^{\prime}$ possessing the above-mentioned properties such that the difference of the sequences defined by the formulas

$$
u_{k}=S\left(u_{k-1}\right)+\zeta_{k}, \quad u_{k}^{\prime}=S\left(u_{k-1}^{\prime}\right)+\zeta_{k}^{\prime}, \quad k \geq 1
$$

converges to zero exponentially fast. This will imply the required assertions about the Markov semi-group $\mathfrak{P}_{k}^{*}$. We emphasize that $\left\{\zeta_{k}\right\}$ and $\left\{\zeta_{k}^{\prime}\right\}$ are not independent.

Proposition 2.3. Under the conditions of Theorem 2.2, there is an integervalued function $N(r) \geq 1$ such that, if (2.3) holds with $N \geq N(B)$, then there is a probability space $(\Omega, \mathcal{F}, \mathbb{P})$ such that for any initial measure $\Lambda \in \mathcal{P}_{1}(\mathbf{H})$ there exists a pair of random sequences $\left(u_{k}, u_{k}^{\prime}\right)$ that are defined on $\Omega$ and possess the following properties:

(i) The initial distribution of $U_{k}=\left(u_{k}, u_{k}^{\prime}\right)$ coincides with $\Lambda$ :

$$
\mathcal{D}\left(U_{0}\right)=\Lambda .
$$


Furthermore, each of the sequences $\left\{\zeta_{k}\right\}$ and $\left\{\zeta_{k}^{\prime}\right\}$ defined by relations (2.5) consists of i.i.d. random variables such that

$$
\mathcal{D}\left(\zeta_{k}\right)=\mathcal{D}\left(\zeta_{k}^{\prime}\right)=\mathcal{D}\left(\eta_{k}\right)
$$

(ii) There is a constant $M>0$, depending only on $B$, and an integer-valued random variable $\ell(\omega) \geq 0$ such that

$$
\begin{gathered}
\mathrm{P}_{N} u_{k}=\mathrm{P}_{N} u_{k}^{\prime} \quad \text { for } \quad k \geq \ell+1, \\
\mathrm{Q}_{N} \zeta_{k}=\mathrm{Q}_{N} \zeta_{k}^{\prime} \quad \text { for } \quad k \geq 1, \\
\left\langle\|\boldsymbol{u}\|^{2}+\left\|\boldsymbol{u}^{\prime}\right\|^{2}\right\rangle_{\ell}^{k} \leq M \quad \text { for } \quad k \geq \ell+1 .
\end{gathered}
$$

(iii) There are positive constants $\alpha$ and $C$ not depending on $\Lambda$ such that

$$
\begin{gathered}
\mathbb{E} e^{\alpha \ell} \leq C\left(1+\mathfrak{m}_{1}(\Lambda)\right), \\
\left|u_{\ell}\right| \vee\left|u_{\ell}^{\prime}\right| \leq 1 .
\end{gathered}
$$

We emphasize that the random variable $\ell(\omega)$ and the constants $\alpha$ and $C$ depend on $N$. The proof of Propositions 2.3, which is given in Section 4, will imply in fact that $U_{k}=\left(u_{k}, u_{k}^{\prime}\right)$ is a Markov chain. We now show that Theorem 2.2 is a simple consequence of Proposition 2.3.

Proof of Theorem 2.2. Step 1. We first show that for any initial measures $\lambda, \lambda^{\prime} \in$ $\mathcal{P}_{1}(H)$ the following inequality holds:

$$
\left\|\mathfrak{P}_{k}^{*} \lambda-\mathfrak{P}_{k}^{*} \lambda^{\prime}\right\|_{\mathcal{L}}^{*} \leq C_{1}\left(1+\mathfrak{m}_{1}(\lambda)+\mathfrak{m}_{1}\left(\lambda^{\prime}\right)\right) e^{-\beta k}, \quad k \geq 1,
$$

where $C_{1}>0$ is a constant not depending on $\lambda$ and $\lambda^{\prime}$. Indeed, let $u_{k}$ and $u_{k}^{\prime}$ be the random sequences constructed in Proposition 2.3 that correspond to the measure $\Lambda=\lambda \otimes \lambda^{\prime}$. Then, in view of (2.6), $u_{0}$ and $u_{0}^{\prime}$ are (independent) random variables whose distributions coincide with $\lambda$ and $\lambda^{\prime}$, respectively. Therefore, for any $f \in \mathcal{L}(H)$ we have

$$
\Delta_{k}(f):=\left|\left(\lambda_{k}, f\right)-\left(\lambda_{k}^{\prime}, f\right)\right| \leq\left|\mathbb{E}\left(f\left(u_{k}\right)-f\left(u_{k}^{\prime}\right)\right)\right|,
$$

where $\lambda_{k}=\mathfrak{P}_{k}^{*} \lambda$ and $\lambda_{k}^{\prime}=\mathfrak{P}_{k}^{*} \lambda^{\prime}$. Let us set $G_{k}=\{\omega \in \Omega: 2 \ell+1 \leq k\}$ and choose $N \geq 1$ so large that $\log \alpha_{N+1} \geq 4(C M+\log C+2)$, where $C$ is the constant in inequality (1.26). It follows from $(2.8)-(2.10),(2.12)$, and Proposition 1.4 that, for $\omega \in G_{k}$, we have

$$
\left|u_{k}-u_{k}^{\prime}\right| \leq\left(C \alpha_{N+1}^{-\frac{1}{4}}\right)^{k-\ell} e^{C M(k-\ell)}\left|u_{\ell}-u_{\ell}^{\prime}\right| \leq e^{-k} .
$$

Furthermore, inequality (2.11) implies that

$$
\mathbb{P}\left(G_{k}^{c}\right) \leq C\left(1+\mathfrak{m}_{1}(\lambda)+\mathfrak{m}_{1}\left(\lambda^{\prime}\right)\right) e^{-\frac{\alpha k}{2}}
$$


Combining $(2.14)-(2.16)$ and setting $\beta=\frac{1 \wedge \alpha}{2}$, we derive

$$
\begin{aligned}
\Delta_{k}(f) & \leq \mathbb{E}\left\{I_{G_{k}}\left|\left(\lambda_{k}, f\right)-\left(\lambda_{k}^{\prime}, f\right)\right|\right\}+\mathbb{E}\left\{I_{G_{k}^{c}}\left|\left(\lambda_{k}, f\right)-\left(\lambda_{k}^{\prime}, f\right)\right|\right\} \\
& \leq\|f\|_{\mathcal{L}} \mathbb{E}\left\{I_{G_{k}}\left|u_{k}-u_{k}^{\prime}\right|\right\}+2\|f\|_{\mathcal{L}^{\mathcal{P}}} \mathbb{P}\left(G_{k}^{c}\right) \\
& \leq C_{1}\|f\|_{\mathcal{L}}\left(1+\mathfrak{m}_{1}(\lambda)+\mathfrak{m}_{1}\left(\lambda^{\prime}\right)\right) e^{-\beta k} .
\end{aligned}
$$

Taking the supremum over all $f \in \mathcal{L}(H)$ satisfying the condition $\|f\|_{\mathcal{L}} \leq 1$, we obtain (2.13).

Step 2. The existence of a stationary measure $\mu \in \mathcal{P}_{1}(H)$ follows from $(2.13)$ and (1.6) by a standard argument (cf. [16, Lemma 1.2]), and therefore we confine ourselves to the proof of uniqueness and inequality (2.4).

Inequality (2.4) implies that a stationary measure is unique in the class $\mathcal{P}_{1}(H)$. Thus, the required uniqueness will be established if we show that any stationary measure belongs to $\mathcal{P}_{1}(H)$. This assertion follows from (1.6) (cf. [23, Theorem 2.2] for the case of white noise). Finally, to prove (2.4), it suffices to take $\lambda^{\prime}=\mu$ in $(2.13)$.

When proving Theorem 2.2, we established the following assertion on a.s. exponential convergence to zero for the difference $\left|u_{k}-u_{k}^{\prime}\right|($ see $(2.15))$.

Corollary 2.4. Let $\Lambda \in \mathcal{P}_{1}(\mathbf{H})$ be an arbitrary initial measure and let $\left(u_{k}, u_{k}^{\prime}\right)$ be the corresponding random sequences constructed in Proposition 2.3. Then

$$
\left|u_{k}-u_{k}^{\prime}\right| \leq e^{-k} \quad \text { for } \quad k \geq 2 \ell(\omega)+1 .
$$

\section{Coupling operator and its properties}

Recall that $\left\{\eta_{k}\right\}$ is the sequence of i.i.d. random variables in (1.2) and denote by $\chi$ their distribution. Condition 2.1 implies that, if $b_{j} \neq 0$ for $j=1, \ldots, N$, then the projection $\chi_{N}=\mathrm{P}_{N} \chi$ is absolutely continuous with respect to the Lebesgue measure $d x$ :

$$
\chi_{N}(d x)=p(x) d x
$$

where we set

$$
p(x)=\prod_{j=1}^{N} q_{j}\left(x_{j}\right), \quad q_{j}\left(x_{j}\right)=b_{j}^{-1} p_{j}\left(x_{j} / b_{j}\right), \quad x=\left(x_{1}, \ldots, x_{N}\right) \in H_{N} .
$$

For any $u \in H$, let $\nu_{u}$ be the distribution of the random variable $\mathrm{P}_{N}\left(S(u)+\eta_{1}\right)$. It follows from (3.1) that $\nu_{u}$ is also absolutely continuous with respect to the Lebesgue measure, and its density has the form $p\left(x-\mathrm{P}_{N} S(u)\right)$.

The following result is a restatement of Lemma 3.2 in [16].

Lemma 3.1. Suppose that Condition 2.1 is satisfied and that $b_{j} \neq 0$ for $j=$ $1, \ldots, N$, where $N \geq 1$ is an arbitrary integer. Then there is a probability space $(\Omega, \mathcal{F}, \mathbb{P})$ such that for any pair of functions $u, u^{\prime} \in H$ there are $H$-valued random variables $\zeta=\zeta\left(u, u^{\prime}, \omega\right)$ and $\zeta^{\prime}=\zeta^{\prime}\left(u, u^{\prime}, \omega\right)$ possessing the following properties: 
(i) The distributions of $\zeta$ and $\zeta^{\prime}$ coincide with $\chi$.

(ii) The random variables $\left(\mathrm{Q}_{N} \zeta, \mathrm{Q}_{N} \zeta^{\prime}\right)$ and $\left(\mathrm{P}_{N} \zeta, \mathrm{P}_{N} \zeta^{\prime}\right)$ are independent, and the projections $\mathrm{Q}_{N} \zeta$ and $\mathrm{Q}_{N} \zeta^{\prime}$ coincide for all $\omega \in \Omega$ and do not depend on $\left(u, u^{\prime}\right)$.

(iii) Let us set

$$
v=\mathrm{P}_{N}(S(u)+\zeta), \quad v^{\prime}=\mathrm{P}_{N}\left(S\left(u^{\prime}\right)+\zeta^{\prime}\right) .
$$

Then the pair $\left(v, v^{\prime}\right)$ is a maximal coupling ${ }^{3}$ for $\left(\nu_{u}, \nu_{u^{\prime}}\right)$. Moreover, there is $C_{N} \geq 1$ depending on $\min \left\{b_{j}: 1 \leq j \leq N\right\}$ such that

$$
\mathbb{P}\left\{v \neq v^{\prime}\right\} \leq C_{N}\left|S(u)-S\left(u^{\prime}\right)\right| \text { for all } u, u^{\prime} \in H .
$$

(iv) The random variables $\zeta$ and $\zeta^{\prime}$ are measurable functions of $\left(u, u^{\prime}, \omega\right) \in$ $H \times H \times \Omega$.

Proof. We only outline the proof. As is shown in [16] (see Lemma 3.2 and inequality (3.8)), the variational distance between $\nu_{u}$ and $\nu_{u^{\prime}}$ admits the estimate

$$
\left\|\nu_{u}-\nu_{u^{\prime}}\right\|_{\mathrm{var}} \leq C_{N}\left|S(u)-S\left(u^{\prime}\right)\right| .
$$

Let $\left(v, v^{\prime}\right)$ be a maximal coupling for $\left(\nu_{u}, \nu_{u^{\prime}}\right)$. By Theorem 4.2 in [16], we can assume that the random variables $v$ and $v^{\prime}$ are defined on the same probability space $\left(\Omega_{1}, \mathcal{F}_{1}, \mathbb{P}_{1}\right)$ for all $u, u^{\prime} \in H$ and are measurable functions of $\left(u, u^{\prime}, \omega_{1}\right)$. Let $\left(\Omega_{2}, \mathcal{F}_{2}, \mathbb{P}_{2}\right)$ be the probability space on which $\eta_{1}$ is given. We denote by $(\Omega, \mathcal{F}, \mathbb{P})$ the direct product of $\left(\Omega_{i}, \mathcal{F}_{i}, \mathbb{P}_{i}\right), i=1,2$, and define $\zeta$ and $\zeta^{\prime}$ by the relations

$$
\begin{gathered}
\mathrm{P}_{N} \zeta(\omega)=v\left(\omega_{1}\right)-\mathrm{P}_{N} S(u), \quad \mathrm{P}_{N} \zeta^{\prime}(\omega)=v^{\prime}\left(\omega_{1}\right)-\mathrm{P}_{N} S\left(u^{\prime}\right) \\
\mathrm{Q}_{N} \zeta(\omega)=\mathrm{Q}_{N} \zeta^{\prime}(\omega)=\mathrm{Q}_{N} \eta\left(\omega_{2}\right)
\end{gathered}
$$

where $\omega=\left(\omega_{1}, \omega_{2}\right) \in \Omega$. Assertions (i), (ii), and (iv) and the fact that $\left(v, v^{\prime}\right)$ is a maximal coupling for $\left(\nu_{u}, \nu_{u^{\prime}}\right)$ follow from the construction, while inequality (3.2) is a consequence of (3.3) and the definition of maximal coupling (see Section 5.3).

Let us define coupling operators by the formulas

$$
\mathcal{R}\left(u, u^{\prime}, \omega\right)=S(u)+\zeta\left(u, u^{\prime}, \omega\right), \quad \mathcal{R}^{\prime}\left(u, u^{\prime}, \omega\right)=S\left(u^{\prime}\right)+\zeta^{\prime}\left(u, u^{\prime}, \omega\right),
$$

where $u, u^{\prime} \in H$ and $\omega \in \Omega$. We shall need the following lemma:

Lemma 3.2. Under the conditions of Lemma 3.1, for any $r>0$ there is $\varepsilon>0$ such that

$$
\mathbb{P}\left\{|\mathcal{R}|+\left|\mathcal{R}^{\prime}\right| \leq\left(\frac{1+q}{2}\left(|u|+\left|u^{\prime}\right|\right)\right) \vee r\right\} \geq \varepsilon \quad \text { for all } u, u^{\prime} \in H .
$$

\footnotetext{
${ }^{3}$ For the definition of a maximal coupling, see Section 5.3.
} 
Proof. Step 1. For $C>0$, we set $A_{C}=\left\{|\zeta|+\left|\zeta^{\prime}\right| \leq C\right\}$ and choose $C$ so large that $\mathbb{P}\left(A_{C}\right) \geq \frac{1}{2}$ for all $u, u^{\prime} \in H$. If $|u|+\left|u^{\prime}\right| \geq R:=\frac{2 C}{1-q}$, where $q$ is the constant in (1.1), then for $\omega \in A_{C}$ we have

$$
|\mathcal{R}|+\left|\mathcal{R}^{\prime}\right| \leq q\left(|u|+\left|u^{\prime}\right|\right)+C \leq \frac{1+q}{2}\left(|u|+\left|u^{\prime}\right|\right) .
$$

Thus, if $|u|+\left|u^{\prime}\right| \geq R$, then (3.5) holds with $\varepsilon=\frac{1}{2}$.

Step 2. We now assume that $|u|+\left|u^{\prime}\right| \leq R$. Suppose that for any $\delta>0$ we found $\varepsilon^{\prime}=\varepsilon^{\prime}(\delta)>0$ such that

$$
\mathbb{P}\left\{|v| \vee\left|v^{\prime}\right| \leq \delta\right\} \geq \varepsilon^{\prime} \quad \text { for } \quad|u|+\left|u^{\prime}\right| \leq R .
$$

It follows from assertion (ii) of Lemma 3.1 and the condition $p_{j}(r)>0$ that

$$
\mathbb{P}\left\{\left|\mathrm{P}_{N} \mathcal{R}\right| \vee\left|\mathrm{P}_{N} \mathcal{R}^{\prime}\right| \leq \delta,\left|\mathrm{Q}_{N} \zeta\right|=\left|\mathrm{Q}_{N} \zeta^{\prime}\right| \leq \delta\right\} \geq \varepsilon \quad \text { for } \quad|u|+\left|u^{\prime}\right| \leq R,
$$

where $\varepsilon=\varepsilon(\delta)>0$ does not depend on $\left(u, u^{\prime}\right)$. Therefore, with probability $\geq \varepsilon$, we have

$$
\begin{aligned}
|\mathcal{R}|+\left|\mathcal{R}^{\prime}\right| & \leq\left|\mathrm{P}_{N} \mathcal{R}\right|+\left|\mathrm{P}_{N} \mathcal{R}^{\prime}\right|+\left|\mathrm{Q}_{N} \mathcal{R}\right|+\left|\mathrm{Q}_{N} \mathcal{R}^{\prime}\right| \\
& \leq 2 \delta+|S(u)|+\left|S\left(u^{\prime}\right)\right|+2\left|\mathrm{Q}_{N} \zeta\right| \\
& \leq q\left(|u|+\left|u^{\prime}\right|\right)+4 \delta
\end{aligned}
$$

Choosing $\delta=\frac{r(1-q)}{8}$, we see that the above expression does not exceed $\left(\frac{1+q}{2}(|u|+\right.$ $\left.\left.\left|u^{\prime}\right|\right)\right) \vee r$. This completes the proof of (3.5) in the case $|u|+\left|u^{\prime}\right| \leq R$.

Step 3. Thus, it remains to establish (3.6). To this end, recall that $\left(v, v^{\prime}\right)$ is a maximal coupling for $\left(\nu_{u}, \nu_{u^{\prime}}\right)$, and therefore, by Lemma 5.2 , we have

$$
\mathbb{P}\left\{|v| \vee\left|v^{\prime}\right| \leq \delta\right\} \geq \mathbb{P}\left\{|v| \leq \delta, v=v^{\prime}\right\}=\nu_{u} \wedge \nu_{u^{\prime}}\left(B_{\delta}^{N}\right),
$$

where $B_{\delta}^{N}=\left\{x \in H_{N}:|x| \leq \delta\right\}$ and $\nu_{u} \wedge \nu_{u^{\prime}}$ is the minimum of $\nu_{u}$ and $\nu_{u^{\prime}}$ (see Section 5.3 for definition). Hence, it suffices to show that for any $\delta>0$ there is $\varepsilon^{\prime}>0$ such that

$$
\nu_{u} \wedge \nu_{u^{\prime}}\left(B_{\delta}^{N}\right) \geq \varepsilon^{\prime} \quad \text { for } \quad|u|+\left|u^{\prime}\right| \leq R .
$$

To prove (3.7), we recall that

$$
\nu_{u}(d x)=p\left(x-\mathrm{P}_{N} S(u)\right) d x, \quad \nu_{u^{\prime}}(d x)=p\left(x-\mathrm{P}_{N} S\left(u^{\prime}\right)\right) d x .
$$

Inequality (3.7) is a simple consequence of these formulas and the fact that $p_{j}(r)>0$ for all $j \geq 1$ and $r \in \mathbb{R}$.

\section{$4 \quad$ Proof of Proposition 2.3}

\subsection{Description of coupled sequences}

Throughout this section, we assume that Condition 2.1 is satisfied, and therefore we can define the coupling operators (3.4). We now use them to construct pairs 
of sequences satisfying (2.5) that correspond to a given initial measure and possess the properties mentioned in Proposition 2.3.

Let $\left(\Omega^{0}, \mathcal{F}^{0}, \mathbb{P}^{0}\right)$ be a probability space such that for any measure $\Lambda \in \mathcal{P}(\mathbf{H})$, $\mathbf{H}=H \times H$, there is a random variable $U=\left(u, u^{\prime}\right)$ on $\Omega^{0}$ whose distribution coincides with $\Lambda$. (For the existence of such a space, see [24, Exercise 1.1.16].) We denote by $\left(\Omega^{k}, \mathcal{F}^{k}, \mathbb{P}^{k}\right), k \geq 1$, independent copies of the probability space constructed in Lemma 3.1 and by $(\Omega, \mathcal{F}, \mathbb{P})$ the direct product of $\left(\Omega^{k}, \mathcal{F}^{k}, \mathbb{P}^{k}\right)$, $k \geq 0$. Points of $\Omega$ will be denoted by $\omega=\left(\omega^{0}, \omega^{1}, \ldots\right)$, where $\omega^{k} \in \Omega^{k}$. Any random variable $\xi\left(\omega^{k}\right)$ can be extended to $\Omega$ by the formula $\xi(\omega)=\xi\left(\omega^{k}\right)$ and regarded as a random variable on $\Omega$.

We now turn to construction of random sequences $\left(u_{k}, u_{k}^{\prime}\right)$. Let $\Lambda \in \mathcal{P}(\mathbf{H})$ be an arbitrary measure and let $U=\left(u, u^{\prime}\right)$ be a random variable on $\Omega^{0}$ with distribution $\Lambda$. We set $u_{0}(\omega)=u(\omega), u_{0}^{\prime}(\omega)=u^{\prime}(\omega)$, and

$$
\begin{aligned}
& u_{k}(\omega)=\mathcal{R}\left(u_{k-1}(\omega), u_{k-1}^{\prime}(\omega), \omega^{k}\right), \\
& u_{k}^{\prime}(\omega)=\mathcal{R}^{\prime}\left(u_{k-1}(\omega), u_{k-1}^{\prime}(\omega), \omega^{k}\right),
\end{aligned}
$$

where $k \geq 1$. Assertion (i) of Proposition 2.3 follows from the construction. Properties (ii) and (iii) will be established in Section 4.3.

\subsection{Auxiliary assertions}

Let us note that formulas (3.4) and (4.1) and the definition of the probability space $(\Omega, \mathcal{F}, \mathbb{P})$ imply that $U_{k}=\left(u_{k}, u_{k}^{\prime}\right)$ is a Markov chain for any initial measure $\Lambda$. We shall denote by $\left(U_{k}, \mathbb{P}_{U}\right), U=\left(u, u^{\prime}\right) \in \mathbf{H}$, the associated Markov family (see the end of Section 1.1). We now study some properties of the family $\left(U_{k}, \mathbb{P}_{U}\right)$.

Let us introduce the stopping time

$$
T(M)=\min \left\{k \geq 1:\left\langle\|\boldsymbol{u}\|^{2}+\left\|\boldsymbol{u}^{\prime}\right\|^{2}\right\rangle_{0}^{k}>M\right\},
$$

where the brackets $\langle\cdot\rangle$ are defined by (1.9) and (1.24). Note that this stopping time is different from the one given by (1.10); however, we use the same notation since they play similar roles for the Markov families $\left(U_{k}, \mathbb{P}_{U}\right)$ and $\left(u_{k}, \mathbb{P}_{u}\right)$. The proof of the following result is similar to that of Proposition 1.2.

Lemma 4.1. There is $\delta>0$ depending only on $B$ such that for any initial measure $\Lambda \in \mathcal{P}(\mathbf{H})$ satisfying the condition

$$
\int_{H} e^{\delta\left(|u|^{2}+\left|u^{\prime}\right|^{2}\right)} \Lambda\left(d u, d u^{\prime}\right) \leq R
$$

where $R>0$ is a constant, we have

$$
\mathbb{P}_{\Lambda}\{T(M)=k\} \leq R Q^{k} e^{-\delta M(k+1)}, \quad k \geq 1 .
$$

In particular, if $\delta M \geq \log (R+Q)$, then

$$
\mathbb{P}_{\Lambda}\{T(M)=\infty\} \geq \frac{R}{R+Q} .
$$


Let $\tau_{d}$ be the first hitting time of the set $\mathbf{B}_{d}=\left\{\left(u, u^{\prime}\right) \in \mathbf{H}:|u| \vee\left|u^{\prime}\right| \leq d\right\}$ (cf. (1.15)):

$$
\tau_{d}=\min \left\{k \geq 0:\left|u_{k}\right| \vee\left|u_{k}^{\prime}\right| \leq d\right\}
$$

Lemma 4.2. For any $d>0$ there are positive constants $\gamma$ and $C$ such that for any initial measure $\Lambda \in \mathcal{P}_{1}(\mathbf{H})$ we have

$$
\mathbb{E}_{\Lambda} e^{\gamma \tau_{d}} \leq C\left(1+\mathfrak{m}_{1}(\Lambda)\right) .
$$

Proof. As was shown in the proof of Proposition 1.3, inequality (4.4) will follow from the two statements below:

(a) There are positive constants $R$ and $C$ such that for any initial measure $\lambda \in \mathcal{P}_{1}(\mathbf{H})$ we have

$$
\mathbb{E}_{\Lambda} q^{-\tau_{R}} \leq C\left(1+\mathfrak{m}_{1}(\Lambda)\right) .
$$

(b) For any $R>0$ and $d>0$ there is an integer $l \geq 1$ and a constant $p>0$ such that

$$
\mathbb{P}_{U}\left\{U_{l} \in \mathbf{B}_{d}\right\} \geq p \quad \text { for any } \quad U=\left(u, u^{\prime}\right) \in \mathbf{B}_{R} .
$$

Proof of (a) is similar to that of Lemma 5.1, and we shall not dwell on it. To prove (b), we use Lemma 3.2. Inequality (3.5) and the Markov property imply that

$$
\mathbb{P}_{\Lambda}\left\{\left|u_{l}\right|+\left|u_{l}^{\prime}\right| \leq\left(c^{l}\left(|u|+\left|u^{\prime}\right|\right)\right) \vee d\right\} \geq \varepsilon^{l} \quad \text { for any } \quad l \geq 1,
$$

where $c=\frac{1+q}{2}<1$ and $\varepsilon=\varepsilon(d)>0$ is a constant not depending on $l$. Choosing the integer $l \geq 1$ so large that $2 R c^{l} \leq d$, we obtain (4.5) with $p=\varepsilon^{l}$.

We now turn to the most important property of the Markov family $\left(U_{k}, \mathbb{P}_{U}\right)$. Let us fix a constant $M>0$ and consider the stopping time

$$
\sigma(M)=T(M) \wedge \min \left\{k \geq 1: \mathrm{P}_{N} u_{k} \neq \mathrm{P}_{N} u_{k}^{\prime}\right\},
$$

where $N$ is the integer entering the definition of the coupling operators $\mathcal{R}$ and $\mathcal{R}^{\prime}$ (see Lemma 3.1). In other words, $\sigma(M)$ is the first instant $k \geq 1$ when either the projections of $u_{k}$ and $u_{k}^{\prime}$ to $H_{N}$ are different or the time average $\left\langle\|\boldsymbol{u}\|^{2}+\left\|\boldsymbol{u}^{\prime}\right\|^{2}\right\rangle_{0}^{k}$ is greater than $M$.

Proposition 4.3. There is a non-decreasing integer-valued function $N(r) \geq 1$ such that the following statements hold as soon as condition (2.3) is satisfied with $N \geq N(B)$ :

(i) Let $C_{N} \geq 1$ be the constant in (3.2) and let $d=\frac{1}{2 C_{N} e^{2}}$. Then there is a constant $M>0$ depending only on $B$ such that

$$
\mathbb{P}_{U}\{\sigma(M)=k\} \leq 2 e^{-2 k}, \quad k \geq 1,
$$

where $U \in \mathbf{B}_{d}$ is an arbitrary initial point. 
(ii) For any $U \in \mathbf{B}_{d}$, we have

$$
\begin{aligned}
\mathbb{P}_{U}\{\sigma(M)=\infty\} & \geq \frac{1}{2}, \\
\mathbb{E}_{U}\left(I_{\{\sigma(M)<\infty\}} e^{\sigma(M)}\right) & \leq 2 .
\end{aligned}
$$

Proof. Inequalities (4.7) and (4.8) follow immediately from (4.6), and therefore we confine ourselves to the proof of (i).

Let $\delta$ and $Q$ be the constants in Lemma 4.1 and Condition 2.1 (see (2.2)), respectively. Without loss of generality, we can assume that $\delta \leq 1$ and $Q \geq e^{2 \delta}$. We set $M=\frac{\log Q+2}{\delta}$ and introduce the events ${ }^{4}$

$$
\begin{gathered}
A(k)=\left\{\mathrm{P}_{N} u_{k}=\mathrm{P}_{N} u_{k}^{\prime}\right\}, \quad \bar{A}(k)=\bigcap_{l=1}^{k} A(l), \\
D(k)=\left\{\left\langle\|\boldsymbol{u}\|^{2}+\left\|\boldsymbol{u}^{\prime}\right\|^{2}\right\rangle_{0}^{k} \leq M\right\} .
\end{gathered}
$$

The proof of (4.6) is by induction on $k$. Let $k=1$. We have

$$
\{\sigma(M)=1\} \subset\{T(M)=1\} \cup A(1)^{c} .
$$

Lemma 4.1 implies that

$$
\mathbb{P}_{U}\{T(M)=1\} \leq Q e^{2 \delta(1-M)} \leq e^{-2} .
$$

Furthermore, since $\left|S(u)-S\left(u^{\prime}\right)\right| \leq|u|+\left|u^{\prime}\right| \leq 2 d$ for $U=\left(u, u^{\prime}\right) \in \mathbf{B}_{d}$, it follows from assertion (iii) of Lemma 3.1 that

$$
\mathbb{P}_{U}\left\{A(1)^{c}\right\}=\mathbb{P}_{U}\left\{\mathrm{P}_{N} u_{k} \neq \mathrm{P}_{N} u_{k}^{\prime}\right\} \leq 2 C_{N} d \leq e^{-2} .
$$

Combining (4.9) - (4.11), we obtain (4.6) with $k=1$.

We now assume that $k=m \geq 2$ and that for $1 \leq k \leq m-1$ inequality (4.6) is already established. It follows from the definition of $\sigma(M)$ that (cf. (4.9))

$$
\{\sigma(M)=m\} \subset\{T(M)=m\} \cup B(m),
$$

where $B(m)=\bar{A}(m-1) \cap A(m)^{c} \cap\{T(M) \geq m\}$. Let us estimate the probability of the events on the right-hand side of (4.12). Lemma 4.1 implies that

$$
\mathbb{P}_{U}\{T(M)=m\} \leq Q^{m} e^{2 \delta-\delta M(m+1)} \leq e^{-(\delta M-\log Q) m} \leq e^{-2 m},
$$

Furthermore, using inequality (4.6) for $1 \leq k \leq m-1$, for any $U \in \mathbf{B}_{d}$ we derive

$$
\mathbb{P}_{U}\{\bar{A}(m-1) \cap D(m-1)\} \geq \mathbb{P}_{U}\{\sigma(M) \geq m\} \geq 1-2 \sum_{k=1}^{m-1} e^{-2 k} \geq \frac{1}{2}
$$

\footnotetext{
${ }^{4}$ Note that, if $M=\frac{\log Q+2}{\delta}$, then inequalities (4.2) and (4.3) hold for any initial measure $\Lambda$ supported by $\mathbf{B}_{1}$.
} 
Therefore, since $\{T(M) \geq m\} \subset D(m-1)$, we can write

$$
\begin{aligned}
\mathbb{P}_{U}(B(m)) & \leq \mathbb{P}_{U}\left\{\bar{A}(m-1) \cap A(m)^{c} \cap D(m-1)\right\} \\
& \leq \mathbb{P}_{U}\left\{\mathrm{P}_{N} u_{m} \neq \mathrm{P}_{N} u_{m}^{\prime} \mid \bar{A}(m-1) \cap D(m-1)\right\} .
\end{aligned}
$$

Now note that, in view of Proposition 1.4, for $\mathbb{P}_{U}$-a.e. $\omega \in \bar{A}(m-1) \cap D(m-1)$, we have

$$
\left|S\left(u_{m-1}\right)-S\left(u_{m-1}^{\prime}\right)\right| \leq 2 d e^{C M m}\left(C \alpha_{N+1}^{-\frac{1}{4}}\right)^{m} .
$$

It follows that, if $\log \alpha_{N+1} \geq 4(C M+\log C+2)$, then

$$
\left|S\left(u_{m-1}\right)-S\left(u_{m-1}^{\prime}\right)\right| \leq 2 d e^{-2 m} .
$$

Combining this inequality with (3.2) and using the Markov property, we see that

$$
\mathbb{P}_{U}\left\{\mathrm{P}_{N} u_{m} \neq \mathrm{P}_{N} u_{m}^{\prime} \mid \bar{A}(m-1) \cap D(m-1)\right\} \leq 2 C_{N} d e^{-2 m} \leq e^{-2 m} .
$$

The required inequality (4.6) with $k=m$ follows now from (4.12) - (4.15).

\subsection{Proof of $(2.8)-(2.12)$}

We first outline the scheme of the proof. Let us fix an integer $N \geq 1$ and positive constants $M$ and $d \leq 1$ such that (4.6) - (4.8) hold and define a sequence of stopping times $\rho_{k}$ by the following rule.

Let $\rho_{0}$ be the first instant when both components of $U_{k}=\left(u_{k}, u_{k}^{\prime}\right)$ hit the ball $B_{d}$. (Note that $\rho_{0}<\infty$ a.s.) Now two cases are possible: either

$$
\mathrm{P}_{N} u_{k}=\mathrm{P}_{N} u_{k}^{\prime}, \quad\left\langle\|\boldsymbol{u}\|^{2}+\left\|\boldsymbol{u}^{\prime}\right\|^{2}\right\rangle_{\rho_{0}}^{k} \leq M \quad \text { for all } k \geq \rho_{0}+1,
$$

and then we set $\rho_{1}=\infty$, or one of the two conditions in (4.16) is violated at a random time $\rho_{1}^{\prime}$, and then we define $\rho_{1}$ as the first instant $\geq \rho_{1}^{\prime}$ when both $u_{k}$ and $u_{k}^{\prime}$ are in $B_{d}$. If $\rho_{1}=\infty$, then $\rho_{k}=\infty$ for all $k \geq 2$. Otherwise, we again consider two cases and define $\rho_{2}$ in exactly the same way as $\rho_{1}$. Continuing this process, we obtain a sequence of stopping times $\rho_{k}$.

Let us note that $\left|u_{\rho_{k}}\right| \vee\left|u_{\rho_{k}}^{\prime}\right| \leq d$ for any $k \geq 0$, and therefore, by (4.7), with probability $\geq \frac{1}{2}$ we have $\rho_{k+1}=\infty$. We shall show that, for a.a. $\omega \in \Omega$, there is an integer $\bar{k}=\bar{k}(\omega) \geq 0$ such that $\rho_{\bar{k}}<\infty$ and $\rho_{\bar{k}+1}=\infty$. This implies that the random variable $\ell(\omega)=\rho_{\bar{k}(\omega)}(\omega)$ is a.s. finite. Relations (2.8), (2.9) and inequalities (2.10), (2.12) follow immediately from the definition of $\ell$ and assertion (ii) of Lemma 3.1, and (2.11) will be established with the help of (4.4), (4.7), and (4.8).

Let us turn to an accurate proof, which is divided into three steps.

Step 1. We fix $N, M$, and $d$ as specified in the beginning of this subsection and, to shorten notation, set $\sigma=\sigma(M)$ and $\tau=\tau_{d}$. Let us introduce the stopping time

$$
\rho=\sigma+\tau \circ \theta_{\sigma}
$$


and note that the sequence $\rho_{k}$ described above can be defined by the formulas

$$
\rho_{0}=\tau, \quad \rho_{k}=\rho_{k-1}+\rho \circ \theta_{\rho_{k-1}}, \quad k \geq 1 .
$$

We first show that

$$
\mathbb{E}_{\Lambda}\left(I_{\left\{\rho_{k}<\infty\right\}} e^{\alpha \rho_{k}}\right) \leq C a^{k}\left(1+\mathfrak{m}_{1}(\Lambda)\right),
$$

where $\Lambda \in \mathcal{P}_{1}(\mathbf{H})$ is an arbitrary initial measure and $a<1$ and $C$ are positive constants not depending on $\Lambda$ and $k$. To this end, we shall need the following lemma, whose proof is given at the end of this section.

Lemma 4.4. Suppose that $N \geq 1, M>0$ and $d \in(0,1]$ are such that $(4.7)$ and (4.8) hold and let $\gamma$ be the constant in (4.4). Then there are positive constants $\alpha \leq \gamma$ and $a<1$ such that

$$
\begin{array}{r}
\mathbb{P}_{U}\{\rho=\infty\} \geq \frac{1}{2}, \\
\mathbb{E}_{U}\left\{I_{\{\rho<\infty\}} e^{\alpha \rho}\right\} \leq a,
\end{array}
$$

where $U$ is any point in $\mathbf{B}_{d}$.

By (4.20) and the SMP, for any integer $m \geq 0$ we have

$$
\begin{aligned}
\mathbb{E}_{\Lambda}\left(I_{\left\{\rho_{m+1}<\infty\right\}} e^{\alpha \rho_{m+1}}\right) & \leq \mathbb{E}_{\Lambda}\left(I_{\left\{\rho_{m}<\infty\right\}} e^{\alpha \rho_{m}} \mathbb{E}_{\Lambda}\left\{I_{\left\{\rho \circ \bar{\theta}_{m}<\infty\right\}} e^{\alpha \rho \circ \bar{\theta}_{m}} \mid \mathcal{F}_{\rho_{m}}\right\}\right) \\
& \leq \mathbb{E}_{\Lambda}\left(I_{\left\{\rho_{m}<\infty\right\}} e^{\alpha \rho_{m}} \mathbb{E}_{U\left(\rho_{m}\right)}\left\{I_{\{\rho<\infty\}} e^{\alpha \rho}\right\}\right) \\
& \leq a \mathbb{E}_{\Lambda}\left(I_{\left\{\rho_{m}<\infty\right\}} e^{\alpha \rho_{m}}\right)
\end{aligned}
$$

where we set $\bar{\theta}_{m}=\theta_{\rho_{m}}$ and used the fact that $U_{\rho_{m}} \in \mathbf{B}_{d}$. Iterating (4.21) and recalling that $\rho_{0}=\tau$, we derive

$$
\mathbb{E}_{\Lambda}\left(I_{\left\{\rho_{k}<\infty\right\}} e^{\alpha \rho_{k}}\right) \leq a^{k} \mathbb{E}_{\Lambda} e^{\alpha \tau}
$$

Since $\alpha \leq \gamma$ (see Lemma 4.4), inequality (4.18) follows from Lemma 4.2.

Step 2. For any $\omega \in \Omega$, let $\bar{k}(\omega)=\sup \left\{k \geq 0: \rho_{k}<\infty\right\}$. We claim that

$$
\mathbb{P}_{\Lambda}\{\bar{k}<+\infty\}=1
$$

where $\Lambda \in \mathcal{P}_{1}(\mathbf{H})$ is an arbitrary initial measure. Indeed, in view of the SMP and (4.19), we have (cf. (4.21))

$$
\mathbb{P}_{\Lambda}\left\{\rho_{k}<\infty\right\}=\mathbb{E}_{\Lambda}\left(I_{\left\{\rho_{k-1}<\infty\right\}} \mathbb{P}_{U\left(\rho_{k-1}\right)}\{\rho<\infty\}\right) \leq \frac{1}{2} \mathbb{P}_{\Lambda}\left\{\rho_{k-1}<\infty\right\}
$$

Iterating this inequality and noting that $\mathbb{P}_{\Lambda}\{\tau<\infty\}=1$ by Lemma 4.2 , we see that

$$
\mathbb{P}_{\Lambda}\left\{\rho_{k}<\infty\right\} \leq 2^{-k} .
$$

Relation (4.22) follows now from the Borel-Cantelli lemma. 
Step 3. We now set

$$
\ell(\omega)=\left\{\begin{array}{lll}
\rho_{\bar{k}(\omega)}(\omega) & \text { if } & \bar{k}(\omega)<\infty, \\
+\infty & \text { if } & \bar{k}(\omega)=+\infty .
\end{array}\right.
$$

It follows from the definition of $\rho_{k}$ and $\bar{k}$ that (2.8), (2.10), and (2.12) hold. Moreover, assertion (ii) of Lemma 3.1 and relation (4.1) imply (2.9). Thus, it remains to establish (2.11).

In view of (4.22) and (4.18), we have

$$
\mathbb{E}_{\Lambda} e^{\alpha \ell} \leq \sum_{k=0}^{\infty} \mathbb{E}_{\Lambda}\left(I_{\{\bar{k}=k\}} e^{\alpha \rho_{k}}\right) \leq \sum_{k=0}^{\infty} \mathbb{E}_{\Lambda}\left(I_{\left\{\rho_{k}<\infty\right\}} e^{\alpha \rho_{k}}\right) \leq C_{1}\left(1+\mathfrak{m}_{1}(\Lambda)\right),
$$

where $C_{1}>0$ is a constant not depending on $\Lambda$, and we used the fact that $a<1$. This completes the proof of Proposition 2.3.

Proof of Lemma 4.4. The definition of $\rho$ implies that $\{\rho=\infty\} \supset\{\sigma=\infty\}$, and therefore (4.19) follows from (4.7).

To prove (4.20), we first show that

$$
\mathbb{E}_{U}\left\{I_{\{\rho<\infty\}} e^{\delta \rho}\right\} \leq A,
$$

where $\delta=\frac{1}{2}(1 \wedge \gamma)$ and $A>0$ is a constant not depending on $U \in \mathbf{B}_{d}$. Indeed, using (4.17) and the SMP, we derive (cf. (4.21))

$$
\begin{aligned}
\mathbb{E}_{U}\left\{I_{\{\rho<\infty\}} e^{\delta \rho}\right\} & =\mathbb{E}_{U}\left\{I_{\{\sigma<\infty\}} e^{\delta \sigma} \mathbb{E}_{U}\left(I_{\left\{\tau \circ \theta_{\sigma}<\infty\right\}} e^{\delta \tau \circ \theta_{\sigma}} \mid \mathcal{F}_{\sigma}\right)\right\} \\
& =\mathbb{E}_{U}\left\{I_{\{\sigma<\infty\}} e^{\delta \sigma} \mathbb{E}_{U_{\sigma}}\left(e^{\gamma \tau}\right)\right\} .
\end{aligned}
$$

By Lemma 4.2,

$$
\mathbb{E}_{U_{\sigma}}\left(e^{\gamma \tau}\right) \leq C\left(1+\left|U_{\sigma}\right|\right)
$$

Substituting this estimate into (4.24) and using Schwarz's inequality and the fact that $\delta \leq \frac{1}{2}$, we obtain

$$
\begin{aligned}
\mathbb{E}_{U}\left\{I_{\{\rho<\infty\}} e^{\delta \rho}\right\} & \leq C \mathbb{E}_{U}\left\{I_{\{\sigma<\infty\}} e^{\frac{\sigma}{2}}\left(1+\left|U_{\sigma}\right|\right)\right\} \\
& \leq C\left(\mathbb{E}_{U}\left\{I_{\{\sigma<\infty\}} e^{\sigma}\right\} \mathbb{E}_{U}\left\{I_{\{\sigma<\infty\}}\left(1+\left|U_{\sigma}\right|\right)^{2}\right\}\right)^{\frac{1}{2}} .
\end{aligned}
$$

Since $U \in \mathbf{B}_{d}$, inequality (4.23) follows from (4.8) and (1.7).

We now prove (4.20). Let us set $\alpha=\varepsilon \delta$, where $\varepsilon>0$ is chosen below. It follows from (4.23) and (4.19) that

$$
\mathbb{E}_{U}\left\{I_{\{\rho<\infty\}} e^{\alpha \rho}\right\} \leq\left(\mathbb{P}_{U}\{\rho<\infty\}\right)^{1-\varepsilon}\left(\mathbb{E}_{U}\left\{I_{\{\rho<\infty\}} e^{\delta \rho}\right\}\right)^{\varepsilon} \leq 2^{-1+\varepsilon} A^{\varepsilon} .
$$

If $\varepsilon>0$ sufficiently small, the right-hand side of this inequality is smaller than 1 . This completes the proof of (4.20). 


\section{Appendix}

\subsection{Proof of Proposition 1.4}

Using a standard technique of the theory of Navier-Stokes equations (e.g., see [4] or $[15$, Section 6$])$, it can be shown that

$$
\left|L^{\frac{1}{4}}\left(v(t)-v^{\prime}(t)\right)\right| \leq C t^{-\frac{3}{4}} \exp \left(C \int_{0}^{t}\left(\|v(s)\|^{2}+\left\|v^{\prime}(s)\right\|^{2}\right) d s\right)\left|v(0)-v^{\prime}(0)\right|,
$$

where $t \geq 0, v(t)$ and $v^{\prime}(t)$ are arbitrary two solutions of the homogeneous NS system, and $C>0$ is a constant not depending on them. Applying inequality (5.1) and recalling relations (1.25), we derive (cf. [15, Section 2.3])

$$
\begin{aligned}
\left|u_{k}-u_{k}^{\prime}\right| & =\left|Q_{N}\left(u_{k}-u_{k}^{\prime}\right)\right|=\left|Q_{N}\left(S\left(u_{k-1}\right)-S\left(u_{k-1}^{\prime}\right)\right)\right| \\
& \leq \alpha_{N+1}^{-\frac{1}{4}}\left|L^{\frac{1}{4}}\left(S\left(u_{k-1}\right)-S\left(u_{k-1}^{\prime}\right)\right)\right| \\
& \leq C \alpha_{N+1}^{-\frac{1}{4}} \exp \left(C \int_{k-1}^{k}\left(\|v\|^{2}+\left\|v^{\prime}\right\|^{2}\right) d s\right)\left|u_{k-1}-u_{k-1}^{\prime}\right| .
\end{aligned}
$$

Iteration of this inequality results in (1.26).

\subsection{An estimate for the hitting time of a large ball}

Lemma 5.1. Let $K=\mathbb{E}\left|\eta_{1}\right|<\infty$ and let a constant $R>0$ be so large that $r:=q R-(1-q)^{-1} K>0$. Then for any initial measure $\lambda \in \mathcal{P}_{1}(H)$ we have

$$
\mathbb{E}_{\lambda} q^{-\tau_{R}} \leq r^{-1} \mathfrak{m}_{1}(\lambda)+1 .
$$

Proof. Derivation of (5.2) is quite standard and is based on the existence of a Lyapunov function.

Step 1. Let us define a martingale $M_{k}$ by the formulas

$$
M_{0}=0, \quad M_{k}=\sum_{l=1}^{k} q^{-l}\left(\left|\eta_{l}\right|-\mathbb{E}\left|\eta_{l}\right|\right), \quad k \geq 1 .
$$

We first show that

$$
r I_{\left\{\tau_{R} \geq 1\right\}} q^{-k \wedge \tau_{R}} \leq\left|u_{0}\right|+M_{k \wedge \tau_{R}}, \quad k \geq 1 .
$$

Indeed, if $\tau_{R}=0$, then the left hand side of (5.3) is zero, whereas the right-hand side is non-negative. Let us assume that $\tau_{R} \geq 1$. Multiplying inequality (1.5) by $I_{\left\{\tau_{R} \geq 1\right\}} q^{-k}$ and replacing $k$ by $\hat{\tau}:=k \wedge\left(\tau_{R}-1\right)$, we derive ${ }^{5}$

$$
q^{-\hat{\tau}} I_{\left\{\tau_{R} \geq 1\right\}}\left|u_{\hat{\tau}}\right| \leq\left|u_{0}\right|+\sum_{l=1}^{\hat{\tau}} q^{-l}\left|\eta_{l}\right|,
$$

\footnotetext{
${ }^{5}$ Note that $\hat{\tau}$ is not a stopping time.
} 
where the sum on the right-hand side is zero if $\hat{\tau}=0$. We now note that

$$
\begin{aligned}
q^{-\hat{\tau}}\left|u_{\hat{\tau}}\right| & \geq q^{-k \wedge \tau_{R}+1} R, \\
\sum_{l=1}^{\hat{\tau}} q^{-l}\left|\eta_{l}\right| & \leq \sum_{l=1}^{k \wedge \tau_{R}} q^{-l}\left|\eta_{l}\right| \leq M_{k \wedge \tau_{R}}+q^{-k \wedge \tau_{R}}(1-q)^{-1} K .
\end{aligned}
$$

Substituting these estimates into (5.4), we obtain (5.3).

Step 2. We now show that $\mathbb{P}_{\lambda}\left\{\tau_{R}<\infty\right\}=1$ for any $\lambda \in \mathcal{P}_{1}(H)$. Indeed, applying $\mathbb{E}_{\lambda}$ to (5.3), we derive

$$
\mathbb{E}_{\lambda}\left(I_{\left\{\tau_{R} \geq 1\right\}} q^{-k \wedge \tau_{R}}\right) \leq r^{-1} \mathfrak{m}_{1}(\lambda)=: C .
$$

By Chebyshev's inequality, we have

$$
\mathbb{P}_{\lambda}\left\{k \wedge \tau_{R} \geq k\right\} \leq C q^{k}, \quad k \geq 1 .
$$

We now note that

$$
\left\{\tau_{R}=\infty\right\}=\bigcap_{k=1}^{\infty}\left\{k \wedge \tau_{R} \geq k\right\}
$$

and therefore inequality (5.6) implies that $\mathbb{P}_{\lambda}\left\{\tau_{R}=\infty\right\}=0$.

Step 3. We can now prove (5.2). Passing to the limit in (5.5) as $k \rightarrow \infty$ and using Fatou's lemma, we derive

$$
\mathbb{E}_{\lambda}\left(I_{\left\{\tau_{R} \geq 1\right\}} q^{-\tau_{R}}\right) \leq r^{-1} \mathfrak{m}_{1}(\lambda) .
$$

It remains to note that $q^{-\tau_{R}} \leq I_{\left\{\tau_{R} \geq 1\right\}} q^{-\tau_{R}}+1$. The proof is complete.

\subsection{A property of maximal coupling}

Let $X$ be a Banach space and let $\nu, \nu^{\prime} \in \mathcal{P}(X)$. A pair of $X$-valued random variables $\left(\xi, \xi^{\prime}\right)$ defined on a common probability space $(\Omega, \mathcal{F}, \mathbb{P})$ is called a coupling for $\left(\nu, \nu^{\prime}\right)$ if $\mathcal{D}(\xi)=\nu$ and $\mathcal{D}\left(\xi^{\prime}\right)=\nu^{\prime}$. A coupling $\left(\xi, \xi^{\prime}\right)$ is said to be maximal if

$$
\mathbb{P}\left\{\xi \neq \xi^{\prime}\right\}=\left\|\nu-\nu^{\prime}\right\|_{\text {var }}:=\sup _{\Gamma \in \mathcal{B}(H)}\left|\nu(\Gamma)-\nu^{\prime}(\Gamma)\right| .
$$

Let $\nu \wedge \nu^{\prime}$ be the minimum of $\nu$ and $\nu^{\prime}$, i.e., a sub-probability measure whose density with respect to $\mu=\nu+\nu^{\prime}$ is equal to $g \wedge g^{\prime}$, where $g=\frac{d \nu}{d \mu}$ and $g^{\prime}=\frac{d \nu^{\prime}}{d \mu}$. For any event $A \in \mathcal{F}$ of positive probability and a random variable $\zeta$, we denote by $\mathcal{D}(\zeta \mid A)$ the conditional distribution of $\zeta$ given $A$.

Lemma 5.2. Let $\left(\xi, \xi^{\prime}\right)$ be a maximal coupling for $\nu, \nu^{\prime} \in \mathcal{P}(X)$. Then

$$
\mathcal{D}\left(\xi \mid \xi=\xi^{\prime}\right)=\mathcal{D}\left(\xi^{\prime} \mid \xi=\xi^{\prime}\right)=\frac{\nu \wedge \nu^{\prime}}{\nu \wedge \nu^{\prime}(X)}
$$


Proof. Let us $\mu=\mathcal{D}\left(\xi \mid \xi=\xi^{\prime}\right)$ and note that

$$
\nu=\mathbb{P}\left\{\xi=\xi^{\prime}\right\} \mu+\mathbb{P}\left\{\xi \neq \xi^{\prime}\right\} \nu_{1}, \quad \nu^{\prime}=\mathbb{P}\left\{\xi=\xi^{\prime}\right\} \mu+\mathbb{P}\left\{\xi \neq \xi^{\prime}\right\} \nu_{1}^{\prime},
$$

where $\nu_{1}$ and $\nu_{1}^{\prime}$ are the distributions of $\xi$ and $\xi^{\prime}$ conditioned on $\left\{\xi \neq \xi^{\prime}\right\}$. It follows that

$$
\nu \wedge \nu^{\prime}=\mathbb{P}\left\{\xi=\xi^{\prime}\right\} \mu+\mathbb{P}\left\{\xi \neq \xi^{\prime}\right\} \nu_{1} \wedge \nu_{1}^{\prime} .
$$

By the definition of a maximal coupling, we have

$$
\mathbb{P}\left\{\xi=\xi^{\prime}\right\}=1-\left\|\nu-\nu^{\prime}\right\|_{\text {var }}=\nu \wedge \nu^{\prime}(X) .
$$

Combining this with (5.8), we see that $\nu_{1} \wedge \nu_{1}^{\prime}=0$. Substitution of this relation into (5.8) results in (5.7).

Acknowledgements. I would like to thank Sergei Kuksin for discussions and encouragement and Sergei Foss for valuable remarks. I am grateful also to the referee who pointed out several inaccuracies in the original version of the paper. The work was supported by EPSRC, grant GR/N63055/01.

\section{References}

[1] A. A. Borovkov, Ergodicity and Stability of Stochastic Processes, John Wiley \& Sons, Chichester, 1998.

[2] J. Bricmont, A. Kupiainen, and R. Lefevere, Ergodicity of the 2D NavierStokes equations with random forcing, Comm. Math. Phys. 224 (2001), 65-81.

[3] J. Bricmont, A. Kupiainen, and R. Lefevere, Exponential mixing for the 2D stochastic Navier-Stokes dynamics, Comm. Math. Phys. 230 (2002), no. $1,87-132$.

[4] P. Constantin and C. Foias, Navier-Stokes Equations, University of Chicago Press, Chicago, IL, 1988.

[5] R. M. Dudley, Real Analysis and Probability, Wadsworth \& Brooks/Cole, Pacific Grove, CA, 1989.

[6] W. E, J.C. Mattingly, and Ya. G. Sinai, Gibbsian dynamics and ergodicity for the stochastically forced Navier-Stokes equation, Comm. Math. Phys. 224 (2001), 83-106.

[7] J.-P. Eckmann and M. Hairer, Uniqueness of the invariant measure for a stochastic PDE driven by degenerate noise, Comm. Math. Phys. 219 (2001), 523-565.

[8] F. Flandoli and B. Maslowski, Ergodicity of the 2-D Navier-Stokes equation under random perturbations, Comm. Math. Phys. 172 (1995), 119-141. 
[9] C. Foiaş and G. Prodi, Sur le comportement global des solutions nonstationnaires des équations de Navier-Stokes en dimension 2, Rend. Sem. Mat. Univ. Padova 39 (1967), 1-34.

[10] R. Z. Has'minskiı̌, Stochastic Stability of Differential Equations, Sijthoff \& Noordhoff, Alphen aan den Rijn, 1980.

[11] M. Hairer, Exponential mixing properties of stochastic PDE's through asymptotic coupling, Probab. Theory Related Fields 124 (2002), no. 3, 345380.

[12] S. Kuksin, On exponential convergence to a stationary measure for nonlinear PDE's, perturbed by random kick-forces, and the turbulence-limit, The M. I. Vishik Moscow PDE seminar, AMS Translations, 2002.

[13] S. Kuksin, Ergodic theorems for 2D statistical hydrodynamics, Rev. Math. Physics 14 (2002), no. 6, 585-600.

[14] S. Kuksin and A. Shirikyan, Stochastic dissipative PDE's and Gibbs measures, Comm. Math. Phys. 213 (2000), 291-330.

[15] S. Kuksin and A. Shirikyan, Ergodicity for the randomly forced 2D NavierStokes equations, Math. Phys. Anal. Geom. 4 (2001), no. 2, 147-195.

[16] S. Kuksin and A. Shirikyan, A coupling approach to randomly forced nonlinear PDE's. I, Comm. Math. Phys. 221 (2001), no. 2, 351-366.

[17] S. Kuksin, A. Piatnitski and A. Shirikyan, A coupling approach to randomly forced nonlinear PDE's. II, Comm. Math. Phys. 230 (2002), no. 1, $81-85$.

[18] S. Kuksin and A. Shirikyan, Coupling approach to white-forced nonlinear PDE's, J. Math. Pures Appl. 81 (2002), 567-602.

[19] N. Masmoudi and L.-S. Young, Ergodic theory of infinite dimensional systems with applications to dissipative parabolic PDEs, Comm. Math. Phys. 227 (2002), 461-481.

[20] J.C. Mattingly, Exponential convergence for the stochastically forced Navier-Stokes equations and other partially dissipative dynamics, Comm. Math. Phys. 206 (1999), no. 2, 273-288.

[21] B. Øksendal, Stochastic Differential Equations, Springer-Verlag, Berlin, 1998.

[22] D. Revuz, Markov Chains, North-Holland, Amsterdam-New York-Oxford, 1984.

[23] A. Shirikyan, Analyticity of solutions for randomly forced two-dimensional Navier-Stokes equations, Russian Math. Surveys 57 (2002), no. 4, 785-799. 
[24] D. W. Stroock, Probability Theory. An Analytic View, Cambridge University Press, Cambridge, 1993.

[25] R. Temam, Navier-Stokes Equations. Theory and Numerical Analysis, North-Holland, Amsterdam-New York-Oxford, 1977.

[26] A. Yu. Veretennikov, Parametric and Non-Parametric Estimation of Markov Chains, Moscow State University Press, Moscow, 2000. (in Russian) 